\title{
Stabilization of breathers in a parametrically driven sine-Gordon system with loss
}

\author{
Grønbech-Jensen, N.; Kivshar, Yu. S.; Samuelsen, Mogens Rugholm
}

Published in:

Physical Review B

Link to article, DOI:

10.1103/PhysRevB.43.5698

Publication date:

1991

Document Version

Publisher's PDF, also known as Version of record

Link back to DTU Orbit

Citation (APA):

Grønbech-Jensen, N., Kivshar, Y. S., \& Samuelsen, M. R. (1991). Stabilization of breathers in a parametrically driven sine-Gordon system with loss. Physical Review B, 43(7), 5698-5701.

https://doi.org/10.1103/PhysRevB.43.5698

\section{General rights}

Copyright and moral rights for the publications made accessible in the public portal are retained by the authors and/or other copyright owners and it is a condition of accessing publications that users recognise and abide by the legal requirements associated with these rights.

- Users may download and print one copy of any publication from the public portal for the purpose of private study or research

- You may not further distribute the material or use it for any profit-making activity or commercial gain

- You may freely distribute the URL identifying the publication in the public portal

If you believe that this document breaches copyright please contact us providing details, and we will remove access to the work immediately and investigate your claim. 


\title{
Stabilization of breathers in a parametrically driven sine-Gordon system with loss
}

\author{
N. Grønbech-Jensen \\ Physics Laboratory I, The Technical University of Denmark, DK-2800 Lyngby, Denmark \\ Yu. S. Kivshar \\ Institute for Low Temperature Physics and Engineering, 47 Lenin Avenue, 310164 Kharkov, U.S.S.R.
}

M. R. Samuelsen

Physics Laboratory I, The Technical University of Denmark, DK-2800 Lyngby, Denmark

(Received 3 May 1990; revised manuscript received 9 November 1990)

\begin{abstract}
We demonstrate that in a parametrically driven sine-Gordon system with loss, a breather, if driven, can be maintained in a steady state at half the external frequency. In the small-amplitude limit the system is described by the effective perturbed nonlinear Schrödinger equation. For an arbitrary breather amplitude the threshold field is determined by a perturbation method and compared with direct numerical simulations. Excellent agreement is found.
\end{abstract}

\section{INTRODUCTION}

The sine-Gordon (sG) system is known to model many physical objects in one-dimensional approximation such as flux flows in long Josephson junctions, dislocations in solids, nonlinear spin waves in the superfluid phases of ${ }^{3} \mathrm{He}$, ferromagnetic or antiferromagnetic systems, etc. The simplest localized (solitons) solutions of the system are divided into two different classes, namely kinks and breathers. The kinks describe motion of topological excitations, for example, flux quanta (fluxons) in long Josephson transmission lines ${ }^{1}$ or various domain walls in magnetic systems. ${ }^{2}$ The other type of soliton solutions, the breather, may be considered as a dynamical bound state of a kink-antikink pair, and it is a nonlinear oscillating state. In real physical systems, when dissipative losses are included, kinks may exist in the form of static configurations, but breather excitations decay away because of the dissipation.

On the other hand, when an ac field is added to the sG system, it can compensate for the dissipative losses and maintain a stationary breather. ${ }^{3-8}$ In the smallamplitude limit, when the sG system is described by an effective nonlinear Schrödinger equation, the problem was first considered by Kaup and Newell, ${ }^{3}$ who found a phase-locked soliton solution by means of perturbation theory. A more detailed investigation reveals a complicated and stochastic dynamics of the ac driven $\mathrm{sG}$ system with loss. ${ }^{4-6}$ The main physically important result of this detailed dynalysis is the following: In the presence of an ac drive force and dissipation a $\mathrm{sG}$ breather can be maintained as a stabilized object. The simple calculation of the threshold field amplitude by means of a perturbative approach is presented in Ref 7 .

In a number of physically important systems, e.g., magnetic systems, an applied field acts on the systems as a parametric force (see, e.g., Ref. 9). In such a case, a threshold generation of linear waves, e.g., the well-known parametric generation of spin waves, ${ }^{9}$ is possible. It is the purpose of this paper to investigate, theoretically, the problem of maintaining a $\mathrm{sG}$ breather in the presence of loss by applying to the system a parametric force oscillating with the double breather frequency. First, we study the problem in the so-called nonlinear Schrödinger (NLS) equation regime, which is valid in the small-amplitude limit. In the more general case, by simple energy arguments, which are similar to those used in Ref. 7, we determine the threshold value of the parametric ac force, and find a value which agrees very well with the value determined by numerical experiments.

\section{SMALL-AMPLITUDE LIMIT}

The equation considered here is the perturbed $\mathrm{sG}$ equation $^{9}$

$$
\phi_{x x}-\phi_{t t}-\sin \phi=\alpha \phi_{t}-\varepsilon_{n} \sin (\omega t) \sin \left(\frac{\phi}{n}\right),
$$

where $\alpha$ is the loss parameter, $\varepsilon_{n}$ is the amplitude of the external parametric force, $\omega$ is its frequency, and $n$ is an integer. Equation (1) arises, for example, as an effective equation of motion for the magnetization vector in magnetic systems, $\phi$ being an angle describing its orientation in a selected plane. The last form of the right-hand side of Eq. (1) describes a variable magnetic field in such a system. In the case of easy-plane ferromagnetics $n=1$, and $\varepsilon_{1}$ has the meaning of the ratio between ac and dc magnetic field amplitudes, $\sin \phi$ being due to the constant part of the magnetic field. Another example is the model of a weak ferromagnetic (antiferromagnetic) system (see, e.g., Ref. 10). In that case the main nonlinearity $(\sim \sin \phi)$ is caused by an anisotropy, and the parameter $\varepsilon_{2}(n=2)$ is the amplitude of the external (variable) magnetic field. Other examples of such parametric forces may be found, e.g., in Ref. 11. The sG breather is a solution of Eq. (1) for $\alpha=\varepsilon_{n}=0$, 


$$
\phi_{\mathrm{br}}^{(0)}(x, t)=4 \tan ^{-1}\left(\frac{\left(1-\omega_{\mathrm{br}}^{2}\right)^{1 / 2} \sin \left(\omega_{\mathrm{br}} t-\theta\right)}{\omega_{\mathrm{br}} \cosh \left[x\left(1-\omega_{\mathrm{br}}^{2}\right)^{1 / 2}\right]}\right),
$$

$\omega_{\mathrm{br}}$ being the breather frequency, $0<\omega_{\mathrm{br}}^{2}<1$, and $\theta$ is an arbitrary initial phase.

If in the steady-state regime the breather Eq. (2) is maintained, all the terms in Eq. (1), except the driving term, contain odd harmonics of $\omega_{\mathrm{br}}$. In order to support the solution, the driving term must also contain odd harmonics of $\omega_{\mathrm{br}}$. To fulfill that we have to consider

$$
\omega=2 \omega_{\mathrm{br}}
$$

i.e., the condition of a parametric generation.

In the small-amplitude limit, $\omega_{\mathrm{br}} \approx 1$, we may look for an oscillating solution to Eq. (1) in the form [cf. Eq. (2) for $1-\omega_{\mathrm{br}}^{2}<<1$ ],

$$
\phi(x, t)=A(x, t) e^{i t}+\text { c.c. },
$$

$A(x, t)$ being a slowly varying envelope. Substituting Eq. (4) into Eq. (1) yields for $A_{t} \ll A$ the perturbed NLS equation,

$$
\begin{aligned}
2 i A_{t}-A_{x x}-\frac{1}{2}|A|^{2} A= & \frac{\varepsilon_{n}}{2 i n}\left[1-\frac{1}{2 n^{2}}|A|^{2}\right] A^{*} e^{i \Omega t} \\
& -i \alpha A
\end{aligned}
$$

where the asterisk means the complex conjugation. The effective driving frequency,

$$
\Omega=\omega-2,
$$

is considered as a small one. Indeed, if the condition Eq. (3) is valid, the external frequency $\omega$ must be $2 \omega_{\mathrm{br}} \approx 2-\mu^{2}$, where $\mu=\left(1-\omega_{\mathrm{br}}^{2}\right)^{1 / 2}$ is the small amplitude of the breather [see Eq. (2)], so that, according to Eq. (6), in the small-amplitude limit $|\Omega| \approx \mu^{2} \ll 1$.

The unperturbed NLS equation $\left(\varepsilon_{n}=\alpha=0\right)$ has a solution in the slowly oscillating form,

$$
A(x, t)=4 i \eta \frac{e^{-2 i \eta^{2} t}}{\cosh (2 \eta x)},
$$

$\eta$ being its parameter. From the viewpoint of the sG system, $\eta=\frac{1}{2}\left(1-\omega_{\mathrm{br}}^{2}\right)^{1 / 2}=\mu / 2$ [cf. Eq. (2)]. To analyze the perturbation-induced dynamics of the oscillating NLS solution Eq. (7) we use a perturbation theory for solitons ${ }^{11}$ considering the perturbative terms as small. According to this approach, we look for a perturbed solution in the form

$$
A(x, t)=4 i \eta(t) \frac{e^{-i \chi(t)}}{\cosh [2 \eta(t) x]},
$$

where $\eta(t)$ and $d \chi(t) / d t$ are slowly varying functions.

Using the so-called adiabatic equations of the perturbation theory for solitons, ${ }^{11}$ we can obtain the equations for the time-dependent soliton parameters $\eta(t)$ and $\psi(t)=2 \chi(t)+\Omega t \quad$ (a similar system was considered in Refs. 12 and 13):

$$
\begin{aligned}
& \frac{d \eta}{d t}=\frac{\varepsilon_{n}}{2 n} \eta\left(1-\frac{16 \eta^{2}}{3 n^{2}}\right) \cos \psi-\alpha \eta, \\
& \frac{d \psi}{d t}=4 \eta^{2}+\Omega-\frac{\varepsilon_{n}}{2 n}\left(1-\frac{16 \eta^{2}}{3 n^{2}}\right) \sin \psi,
\end{aligned}
$$

where

$$
\psi \equiv 2 \chi(t)+\Omega t
$$

Equations (9) and (10) have fixed-point solutions at $\psi=\psi_{*}$ and $\eta=\eta_{*}$, where $\eta_{*}$ and $\psi_{*}$ are solutions of the system

$$
\begin{aligned}
& \frac{\varepsilon_{n}}{2 n}\left[1-\frac{16 \eta^{2}}{3 n^{2}}\right] \cos \psi=\alpha, \\
& 4 \eta^{2}+\Omega-\frac{\varepsilon_{n}}{2 n}\left[1-\frac{16 \eta^{2}}{3 n^{2}}\right) \sin \psi=0 .
\end{aligned}
$$

In the lowest approximation in $\eta^{2}$ we obtain two such solutions analytically $(\Omega<0)$ :

$$
\begin{aligned}
& \eta_{*}^{2}=\frac{|\Omega|}{4} \pm \frac{1}{4}\left[\frac{\varepsilon_{n}^{2}}{4 n^{2}}-\alpha^{2}\right]^{1 / 2}, \\
& \psi_{*}= \pm \arccos \left(\frac{2 \alpha n}{\varepsilon_{n}}\right) .
\end{aligned}
$$

These solutions correspond to nonpropagating solutions whose amplitudes and phases are fixed but positions in space remain arbitrary. Stability analysis of Eqs. (9) and (10) result in the condition $\sin \psi_{*}>0$ for stability. Thus one soliton [Eqs. (12) and (13) $(+)$ ] is stable while the other [Eqs. (12) and (13) (-)] is unstable. Strictly speaking the stable soliton is only marginally stable since the perturbed system Eq. (5) is still translationally invariant. Any perturbation which breaks this invariance may induce soliton motion.

The resulting stabilized soliton has the form

$$
\phi(x, t) \cong \frac{8 \eta_{*} \sin \left(\frac{1}{2} \psi_{*}-\frac{1}{2} \omega t\right)}{\cosh \left(2 \eta_{*} x\right)},
$$

where the soliton parameters $\psi_{*}$ and $\eta_{*}$ are determined by Eqs. (12) and (13). Therefore, the soliton equation (14) is stabilized at one-half the external frequency (i.e., it is a so-called parametrically phase-locked state) by the parametric force provided

$$
\varepsilon_{n}>2 n \alpha \text {. }
$$

\section{ENERGY-BALANCE APPROACH}

To calculate the threshold amplitude of the parametric field allowing a steady-state oscillating breather in the general case, we use the approach proposed in Ref. 7. The method uses energy considerations, and the assumption that the breather mode of the perturbed $\mathrm{sG}$ system, $\phi_{\mathrm{br}}(x, t)$, may be chosen in the form of Eq. (2). A similar approach was also applied to the kink motion in the perturbed sG equation (see, e.g., Ref. 14).

Let us define the energy of the sG system as 


$$
H \equiv \int_{-\infty}^{\infty} d x\left[\frac{1}{2} \phi_{x}^{2}+\frac{1}{2} \phi_{t}^{2}+(1-\cos \phi)\right]
$$

For the pure sG breather equation (2), this energy is $H_{\mathrm{br}}=16\left(1-\omega_{\mathrm{br}}^{2}\right)^{1 / 2}$. In the perturbed sG system Eq. (1) yields

$$
\frac{d H}{d t}=-\alpha \int_{-\infty}^{\infty} \phi_{t}^{2} d x+\varepsilon_{n} \sin (\omega t) \int_{-\infty}^{\infty} \phi_{t} \sin \left[\frac{\phi}{n}\right] d x .
$$

The change in the system energy during one breather period is then found by integrating Eq. (17) over one breather period:

$$
\begin{aligned}
\Delta H & =\int_{0}^{2 \pi / \omega_{\mathrm{br}}} d t \frac{d H}{d t} \\
& =\varepsilon_{n} I_{n}^{(\mathrm{in})} \cos (2 \theta)-\alpha I^{(\text {loss })},
\end{aligned}
$$

where the integrals for the breather energy loss $I^{\text {(loss) }}$, and the energy input to the breather, $I_{n}^{(i n)}$, must be calculated at $\omega=2 \omega_{\text {br }}$ [see, Eq. (3)]:

$$
\begin{aligned}
I^{(\text {loss })} & =\int_{0}^{2 \pi / \omega_{\mathrm{br}}} d t \int_{-\infty}^{\infty} d x\left(\phi_{\mathrm{br}, t}^{(0)}\right)^{2} \\
& =32 \pi \sin ^{-1}\left(1-\omega_{\mathrm{br}}^{2}\right)^{1 / 2}
\end{aligned}
$$

and

$$
I_{n}^{(\mathrm{in})}=\int_{0}^{2 \pi / \omega_{\mathrm{br}}} d t \sin \left(2 \omega_{\mathrm{br}} t\right) \int_{-\infty}^{\infty} d x \phi_{\mathrm{br}, t}^{(0)} \sin \left(\phi_{\mathrm{br}}^{(0)} / n\right) .
$$

A power balance is established between energy input and output when the change in the system energy is zero over one breather period. By putting Eq. (18) to zero, we find the balance condition

$$
\frac{\varepsilon_{n}}{\alpha} \cos (2 \theta)=\frac{I^{\text {(loss) }}}{I_{n}^{(\text {in })}} .
$$

Here, the phase $\theta$ is adjusted to balance the energy input and output of the driven breather. Close to threshold we should have maximum energy input from Eq. (20) and, therefore, close to the threshold the phase of the breather should be taken at $\theta=0$.

We have not been able to calculate the integrals [Eq. (20)] analytically for any value of $n$, but it is possible to do a linear combination of two of the integrals [Eq. (20)]:

$$
\frac{1}{2} I_{1}^{(\mathrm{in})}+I_{2}^{(\mathrm{in})}=16 \pi\left(1-\omega_{\mathrm{br}}^{2}\right)^{1 / 2} .
$$

If the breather frequency is close to the limit one, $\omega_{\mathrm{br}} \rightarrow 1$, we can find approximate expression for the energy input as a first-order approximation around the frequency $\omega_{\mathrm{br}}^{2}=1$,

$$
I_{n}^{(\mathrm{in})} \cong 16 \pi \frac{\left(1-\omega_{\mathrm{br}}^{2}\right)^{1 / 2}}{n \omega_{\mathrm{br}}^{2}}\left[1-\frac{1-\omega_{\mathrm{br}}^{2}}{\omega_{\mathrm{br}}^{2}}\left[1+\frac{2}{n^{2}}\right] \frac{4}{9}\right],
$$

and in this limit the threshold condition equation (21), has the form

$$
\frac{\varepsilon_{n}}{n \alpha} \cong 2-\frac{1}{18}\left[14-\frac{32}{n^{2}}\right]\left(1-\omega_{\text {br }}^{2}\right) .
$$

It is important to note that only the first term in Eq. (23) may be obtained in the framework of the NLS regime considered above [see Eq. (15)]. Taking into account the terms $\left(\sim \eta^{2}\right)$ in Eqs. (9) and (10), we cannot obtain the correct second term of Eq. (23) because in this approximation one needs to take into account the higher-order nonlinear term $\left(\sim|A|^{4} A\right)$ on the left-hand-side of Eq. (5), and the higher-order harmonics.

\section{NUMERICAL SIMULATIONS}

In order to check some of our assumptions and our theoretical results we have performed numerical experiments on the system [Eq. (1)] and numerical calculations

(a)

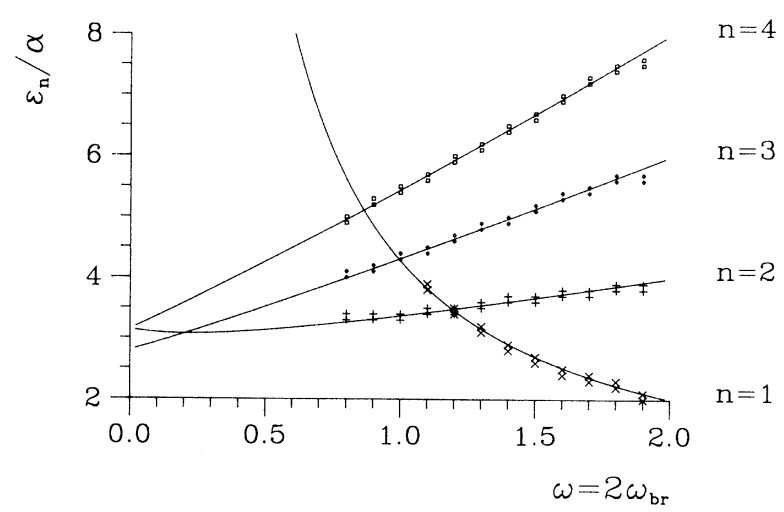

(b)

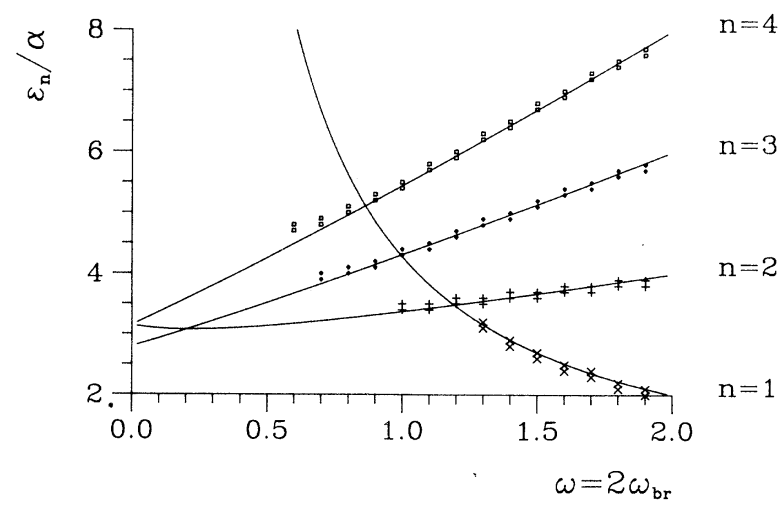

FIG. 1. The threshold parametric force $\epsilon_{n}$ vs the applied external frequency $\omega$ for various values of the loss parameter $\alpha$, (a) $\alpha=0.1$ and (b) $\alpha=0.2$. Shown is $\epsilon_{n} / \alpha$. The solid curves are the perturbative results Eq. (21) for different $n$ with the input energy integrals Eq. (20) calculated numerically at $\theta=0$. The markers on the pictures are the results of the numerical integration of the partial differental equations Eq. (1). 
of the integrals [Eq. (20)] for $\theta=0$. Solving Eq. (1) we have used a second-order implicit finite-difference method. The space and time grid size were chosen to be $\Delta x=0.05$ and $\Delta t=0.02$ of the normalized units. The breather $\phi_{\mathrm{br}}^{(0)}(x, t=0)$ is placed at $x=0$, since the solution is symmetric around this point and we have imposed the boundary conditions to be $\phi_{x}(0)=\phi_{x}(L)=0$. The system length was chosen to be $L=24$ for breather frequencies larger than 0.5 and $L=12$ for breather frequencies less than 0.5 . These choices of system lengths were found to be appropriate to simulate the infinite length of the system. To eliminate the transients, integration of Eq. (1) was performed up to $t=18\left(2 \pi / \omega_{\mathrm{br}}\right)$ in the normalized time units used in Eq. (1), and then the maximum value of $\phi(x=0, t)$ was found in the time interval $18 T<t<20 T, T=2 \pi / \omega_{\mathrm{br}}$ being the period of the breather oscillations. If this maximum differs by more than $50 \%$ from the amplitude of the unperturbed breather $\phi_{\mathrm{br}}^{(0)}(x, t)$, we define the breather to be lost, otherwise we define it to be maintained. A check was also made to ensure that a maintained breather had not turned into a kink-antikink pair.

Figures 1 and 2 show our numerical results. Figure 1 compares the perturbation-theory results with the results of the direct numerical solution of Eq. (1), (a) for $\alpha=0.1$ and (b) for $\alpha=0.2$. The solid curves represent the perturbation results equation (21) with the integrals equation (20) calculated numerically for different values of $n$ and the markers represent the direct numerical integration of Eq. (1). The markers appear in pairs to indicate the resolution of the results of the numerical simulations. A spacing of $0.1 \alpha$ was used as a step of the amplitude $\varepsilon_{n}$. The upper marks of the pair represent the cases where the breathers were kept and the lower marks represent the cases where the breathers were lost. The results are in excellent agreement. For low frequencies the breather could not be kept for any tried value of $\varepsilon_{n}$. We also note that, unlike the case of the ac driving force studied in Refs. 7 and 8 , the numerical results presented do not de-

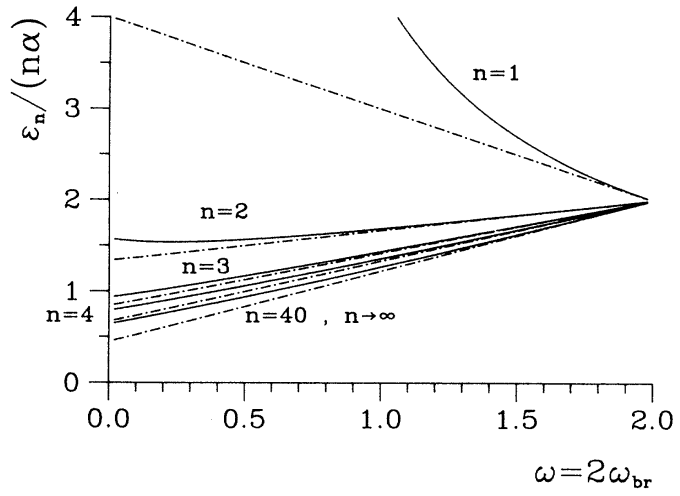

FIG. 2. The comparison of the threshold function $\epsilon_{n} /(n \alpha)$ calculated numericlaly from Eq. (21) (solid lines) and the tangents $\omega_{\mathrm{br}} \rightarrow 1$ [Eq. (23)] obtained analytically (dash-dotted lines).

pend strongly on the value of the dissipative loss [cf. Figs. $1(\mathrm{a})$ and $1(\mathrm{~b})$.

Figure 2 shows a comparison of the functions $\varepsilon_{n} /(n \alpha)$ obtained numerically with the help of Eq. (21) and the tangents calculated analytically in Eq. (23). All these curves tend to the limit value $\varepsilon_{n}=2 n \alpha$, which is also obtained in the NLS-equation approach.

\section{CONCLUDING REMARKS}

We have demonstrated that the sine-Gordon breather can be stabilized by a parametric driver at one-half the external frequency. The problem is investigated analytically in the so-called nonlinear Schrödinger regime, which is valid in the small-amplitude limit. We have used also a perturbation method on energy-flow balance to predict the threshold value of the parametric field for maintaining persistent breather oscillations of nonsmall amplitudes. We find very good agreement with the numerical simulations.
${ }^{1}$ A. Barone and G. Paterno, Physics and Applications of the Josephson Effect (Wiley, New York, 1982).

${ }^{2}$ A. M. Kosevich, B. A. Ivanov, and A. S. Kovalev, Nonlinear Magnetization Waves. Dynamical and Topological Solitons (Naukova Dumka, Kiev, 1983) (in Russian); Phys. Rep. (to be published).

${ }^{3}$ D. J. Kaup and A. C. Newell, Phys. Rev. B 18, 5162 (1978).

${ }^{4}$ A. R. Bishop, K. Fesser, P. S. Lomdahl, and S. E. Trullinger, Physica D 7, 259 (1983).

${ }^{5}$ A. Mazor, A. R. Bishop, and D. W. McLaughlin, Phys. Lett. A 119, 273 (1986)

${ }^{6}$ A. Mazor and A. R. Bishop, Physica D 27, 269 (1987).

${ }^{7}$ P. S. Lomdahl and M. R. Samuelsen, Phys. Rev. A 34, 664 (1986).

${ }^{8}$ P. S. Lomdahl and M. R. Samuelsen, Phys. Lett. A 128, 427
(1988).

${ }^{9}$ A. I. Akhiezer, V. G. Bar'yakhtar, and S. V. Peletminsky, Spin Waves (Nauka, Moscow, 1967) (English translation: NorthHolland, Amsterdam, 1968).

${ }^{10}$ V. G. Bar'yakhtar, B. A. Ivanov, and A. A. Sukstausky, Zh. Eksp. Teor. Fiz. 78, 1509 (1980) [Sov. Phys. JETP 51, 757 (1980)].

${ }^{11}$ Yu. S. Kivshar and B. A. Malomed, Rev. Mod. Phys. 61, 763 (1989).

${ }^{12}$ Yu. S. Kivshar (unpublished).

${ }^{13}$ M. M. Bogdan, A. M. Kosevich, and I. V. Manzhos, Fiz. Nizk. Temp. 11, 991 (1985) [Sov. J. Low Temp. Phys. 11, 547 (1985)].

${ }^{14}$ O. H. Olsen and M. R. Samuelsen, Phys. Rev B 28, 210 (1983); 33, 595 (1986). 